\title{
The Effectiveness of 448-kHz Capacitive Resistive Monopolar Radiofrequency for Subcutaneous Fat Reduction in a Porcine Model
}

\author{
Tae-Rin Kwon ${ }^{1 *}$ \\ Sung-Eun Lee ${ }^{1,2 *}$ \\ Jong Hwan Kim ${ }^{1}$ \\ Yong Jae Jeon ${ }^{4}$ \\ You Na Jang ${ }^{1,2}$ \\ Kwang $\mathrm{Ho} \mathrm{YoO}^{3}$ \\ Beom Joon Kim ${ }^{1,2}$
}

\footnotetext{
${ }^{1}$ Department of Dermatology, Chung-Ang University College of Medicine, Seoul, Korea ${ }^{2}$ Department of Medicine, Graduate School, Chung-Ang University, Seoul, Korea

${ }^{3}$ Department of Dermatology, College of Medicine, Catholic Kwandong University, International St. Mary's Hospital, Incheon, Korea ${ }^{4}$ K1MED Co., Ltd. 205, 206, 207, 209 Woolim e-Biz Center II, Seoul, Korea

*These authors should be considered co-first authors.
}

\author{
Background and Objectives \\ The effectiveness of many physiotherapy modalities in reducing \\ subcutaneous fat has been investigated in numerous previous studies. \\ However, to the best of our knowledge, there have been no attempts to \\ determine the effectiveness of physiotherapy modalities in body \\ contouring. The present report determined the effect of $448-\mathrm{kHz}$ \\ capacitive resistive monopolar radiofrequency (CRMRF) in a porcine \\ model.
}

\section{Materials and Methods}

This study investigated the effect of selective destruction of the subcutaneous fat layer in abdominal fat tissue using CRMRF. The effects of two types of CRMRF (capacitive electric transfer (CET) and resistive electric transfer (RET)] treatment were evaluated using regular digital photography in addition to thermal imaging evaluation, ultrasound measurement, hematological evaluation, and histologic analyses (H\&E (hematoxylin and eosin), Oil red 0 , and immunohistochemistry staining).

\section{Results}

Preclinical evaluation was performed to obtain the data for comparison of the safety and efficacy of the subcutaneous fat reduction after applying CRMRF using CET and RET. After treatment, the thermal transmission was effective, and a $42-47^{\circ} \mathrm{C}$ temperature change was observed in the fat layer while an approximately temperature of $42^{\circ} \mathrm{C}$ was confirmed on the skin surface. Moreover, after the application of both types of CRMRF treatment, fibrotic septa were observed in the adipose tissue induced by heat at the treatment sites. TUNEL staining was also performed to confirm the process of apoptosis in the adipocytes.

\section{Conclusion}

These results suggest that both CET and RET for CRMRF treatment are safe and effective for subcutaneous fat reduction in a porcine model.

\section{Key words}

Radiofrequency; Hyperthermia; Cellular biostimulation; Subcutaneous fat reduction; Apoptosis

\author{
Correspondence \\ Beom Joon Kim \\ Dept. of Dermatology, Chung-Ang University \\ Hospital, 224-1 Heukseok-dong, Dongjak-gu, \\ Seoul 06973, Korea \\ Tel.: +82-2-6299-1525 \\ Fax: +82-2-823-1049 \\ E-mail: beomjoonđunitel.co.kr \\ (c) Korean Society for Laser Medicine and Surgery \\ (c) This is an open access article distributed under the \\ terms of the Creative Commons Attribution Non- \\ Commercial License (http://creativecommons.org/ \\ licenses/by-nc/4.0) which permits unrestricted non- \\ commercial use, distribution, and reproduction in any \\ medium, provided the original work is properly cited.
}




\section{INTRODUCTION}

Variations in fat accumulation patterns lead to differential contour changes along the human body. ${ }^{1}$ These contour changes are related to a complex combination of factors, including lifestyle, age, gender, hormone levels, and genetic elements. ${ }^{2}$ Fat accumulation in the human body has dramatically increased in the past few decades and is associated with many diseases and health problems. ${ }^{3}$ Consequently, research efforts to develop a more effective and safe method for fat reduction are steadily increasing. ${ }^{4}$ Successful selective fat reduction has been reported using a variety of novel methods, including cryolipolysis, ${ }^{5}$ high-intensity focused thermal ultrasound (HIFU), radiofrequency ablation, ${ }^{7}$ and low-level external laser therapy. ${ }^{8}$

A radiofrequency (RF) stimulator is a device used to achieve a medical or cosmetic effect by applying RF to human tissue, converting the tissue to thermal energy and raising the temperature inside the body. ${ }^{9}$ RF stimulators have been reported to increase the temperature, dilate blood vessels, increase blood flow, and relieve pain. ${ }^{10}$ The operation mechanism of this device involves the application of an RF current to tissue to generate thermal energy, and the RF energy converted to thermal energy increases the temperature of the tissue to dilate the blood vessels and increase the blood flow in the tissue, thereby relieving pain in the affected area. ${ }^{11}$ These treatments were recently developed and physical therapy devices le.g., helium and infrared lamps, thermal therapy devices for compressing and applying a hot pack to the affected area, and RF stimulators using RF waves) have been widely used of late. ${ }^{12,13}$

RF typically refers to radio transmission frequency (300$1,200 \mathrm{kHz}$, but RF in treatment devices refers to an RF current. The general commercial alternating current (AC) uses a $60-\mathrm{Hz}$ frequency, whereas treatment devices use frequencies of $300-\mathrm{kHz}$ to tens-of-MHz. That is, RF treatment produces a medical or cosmetic effect by applying an RF current to human biological tissue and harnessing the frictional heat generated by the ions in the cells between the anode and the cathode. ${ }^{14}$

When heat is transmitted to the core at an average of $38-45^{\circ} \mathrm{C}$ through repeated and continuous use of RF, the blood vessels are dilated, which increases the metabolic function, thereby doubling the self-healing ability of the human body. In addition, the process improves the blood and lymphatic circulation, exerts a sterilization effect due to the high temperature, and induces the production of collagen in the dermal layer of the skin. The heat also loosens knotted muscle tissues. ${ }^{15}$ Heat treatment is widely known to have excellent effects on obesity, pore reduction, wrinkle removal, hair promotion, pain relief, etc. 16,17

With regard to the conventional RF stimulator, the RF generator generates the desired frequency, and the electrode pad, which is several times larger than the electrode (typically about 10 times), is attached to a large area like the back or the belly of the human body. An electrode emitting RF energy is attached to the site that requires medical or beauty treatment. The practitioner attaches the electrode pad or the electrode to the body, and the electrode is placed in contact with the treatment site for 10-30 min. ${ }^{18}$ Heat transfer is carried out in two ways. Resistive electric transfer (RET) utilizes the principle that the tissue between the conductor and the counter electrode generates bioheat by accelerating the electronic activity from the center. It is a deep-heat method that can be applied to subcutaneous and visceral fat. Capacitive electric transfer (CET), on the other hand, utilizes the principle that bioheat is generated by active electronic activity centered on the conductor in contact with the skin, and it contributes to the detoxification of the tissue by acting on specific adipocytes to activate venous blood circulation. ${ }^{19,20}$

The main aims of this study were to evaluate the mechanisms of action, efficacy, and safety of the $448-\mathrm{kHz}$ capacitive resistive monopolar radiofrequency (CRMRF) in a porcine model. We found that the technique effectively resulted in fat reduction in the treated areas.

\section{MATERIALS AND METHODS}

\section{Animals and experimental design}

Yorkshire pigs ranging in weight from 40 to $50 \mathrm{~kg}$ were purchased from CRONEX Co., Ltd. (Hwasung, South Korea). The animals were bred in SPF under optimal environmental conditions (temperature: $22 \pm 3^{\circ} \mathrm{C}$; humidity: $50 \pm 10 \%$; cleanliness: 1,500; ventilation: 15T/HR; internal pressure: $3 \mathrm{mmHg}$; airflow speed: $5 \mathrm{~cm} / \mathrm{sec}$; noise: $10 \mathrm{~dB}$ ) and were evaluated in a GLP facility. After checking for the presence of abnormalities and the weight of the pigs, $3 \mathrm{ml}$ of a solution containing Zoletil 50 (tiletamine hydrochloride + zolazepam hydrochloride, Virbac S.A., France) and Rompun (xylazine hydrochloride, Bayer) at a 6:4 ratio was injected intramuscularly for general anesthesia, and then the pigs were moved to the operating room. After they were laid on the operating table, they were intravenously injected with $2 \mathrm{ml}$ of the same solution at the same volume. The airway was secured using a laryngoscope, and an 8.5-mm intubation tube was inserted, followed by 
respiratory anesthesia by administering a 2:1 mixture gas of TERRELL solution lisoflurane, Piramal Critical Care, Inc.) and oxygen. The experiment was then conducted, after which $0.2 \mathrm{ml} / \mathrm{kg}$ of Baytril (enrofloxacin, Komipharm International Co. Ltd.) was injected intramuscularly. After shaving the hair on the pigs' back areas, each back area was subdivided and irradiated using 448-kHz CRMRF (K1MED Co. Ltd, Seoul, Korea) with RET or CET. Radiation was performed after installing the grounding connection on the lower part of the pig's abdomen following sufficient and even application of RF-exclusive lotion for $10 \mathrm{~min}$ (i.e., massaging the target areas) (Fig. $1 \mathrm{~A}$ and B). CRMRF was applied with an RF power from 150 to $200 \mathrm{~W}$ for CET and 80 to $100 \mathrm{~W}$ for RET. All procedures involving animals were conducted in accordance with the guidelines of the Institutional Animal Care and Use Committee of CRONEX Co., Ltd in Korea (No. 201712003).

\section{Serum lipid analysis}

The pigs were restrained manually for collection of blood from the caudal portion of the external jugular vein. Blood samples were centrifuged at $1500 \mathrm{~g}$ for $25 \mathrm{~min}$, and the serum or plasma was removed and stored at $-20^{\circ} \mathrm{C}$ until the time of sample analysis. Serum was collected

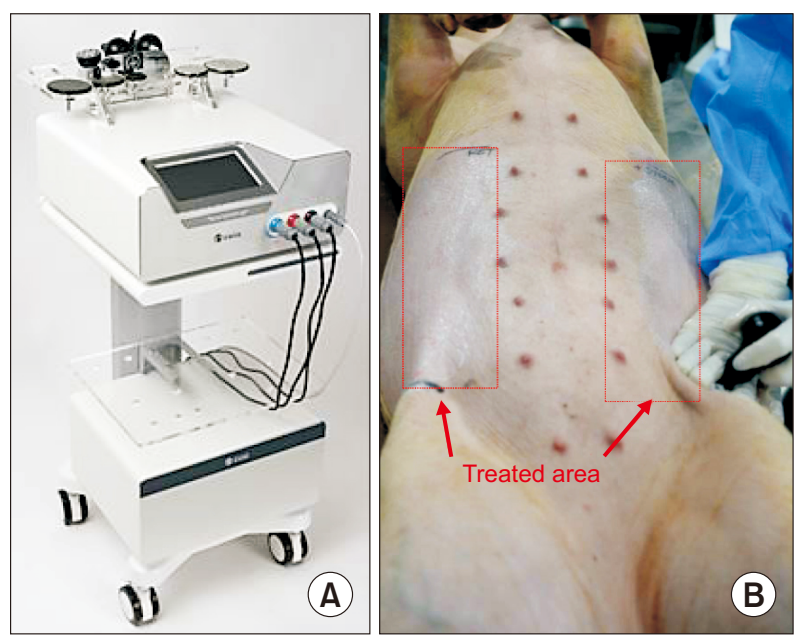

Fig. 1. A 448-kHz CRMRF applicator for subcutaneous fat reduction in a porcine model. (A) This study was conducted to obtain data on the safety and performance of the test device for subcutaneous fat reduction, and a comparative test was conducted when the CRMRF (RET and CET types) of SNE-7100 provided by K1MED Co., Ltd. was combined with STONE. (B) Illustration of the treatment process with the test device during preclinical evaluation of the pig model. The nonclinical study was conducted as a 90-day, single-center, study-comparative group, and parallel preclinical study. Results were evaluated by collecting the data at baseline (0), immediately after application, and on days $1,7,15,30,60$, and 90 . into heparinized tubes (Vacutainer, Becton-Dickinson, CA, USA) containing sodium heparin (143 USP units per tubel and immediately placed on ice. All samples were collected after an overnight fast of at least $12 \mathrm{~h}$. Fasting triglyceride (TG) and cholesterol levels were determined enzymatically. Very-low and low-density lipoproteins (VLDL and LDL, respectively) were precipitated from the plasma using $4 \%$ sodium phosphotungstate, and highdensity lipoprotein (HDL) cholesterol was measured in the supernatant after centrifugation at $1500 \mathrm{~g}$ for $30 \mathrm{~min}$ at $4^{\circ} \mathrm{C} .^{21}$

\section{Clinical evaluation}

Clinical symptoms were measured using images captured daily with a digital camera (Canon 3000D, Canon Inc., Tokyo, Japan). After treatment, macroscopic evaluation of burns and inflammation was performed by digital image analysis, using a phototrichogram system (Folliscope, Lead-M, Seoul, Koreal. The fat layer thickness measurements were evaluated using diagnostic ultrasound (Sonon 300C, Healcerion, Seoul, South Korea) 90 days posttreatment.

\section{Biopsy specimens and histologic measurements}

Paired 8-mm punch biopsy specimens were obtained from each side of the abdominal tissue at baseline and at the end of treatment. Posttreatment biopsy specimens were collected near the previous biopsy site. Tissue samples were fixed in $10 \%$ buffered formalin and embedded in paraffin. Subsequently, 5- $\mu$ m-thick sections, cut using a microtome, were transferred to ProbeOn Plus slides (Fisher Scientific, Pittsburg, PA, USA) and stained with hematoxylin and eosin (H\&E). The skin biopsy samples were stored at $-80^{\circ} \mathrm{C}$ and placed in a Cryomold with optimum cutting temperature (OCT) (Tissue-Tek, Sakura Finetek Inc., Torrance, CA, USA). Next, 10-lm-thick sections were cut using a microtome, placed on slides, and stained with Oil Red 0 , followed by counterstaining of the nuclei with hematoxylin.

\section{TUNEL Iterminal deoxynucleotidyl transferase dUTP nick end labeling) staining}

For the TUNEL experiment, an ApopTag ${ }^{\circledR}$ Peroxidase In-Situ Apoptosis Detection Kit (S7100, Millipore, USA) was used. First, a 3 - $\mu$ m-thick paraffin block was made, and then heat was applied to it at $60^{\circ} \mathrm{C}$ for $1 \mathrm{~h}$ to remove the paraffin, followed by dehydration by washing with $x y-$ lene, 100 and $95 \%$ ethanol, and phosphate-buffered saline (PBS). The tissue sections were treated with proteinase $\mathrm{K}$ $\left(20 \mathrm{\mu g} / \mathrm{ml}\right.$ ) for $15 \mathrm{~min}$ as well as with $3 \% \mathrm{H}_{2} \mathrm{O}_{2}$ to prevent 
peroxidase activity. The slides were then washed with water, immersed in PBS solution for $5 \mathrm{~min}$, and reacted for $2 \mathrm{~min}$ by dropping them into equilibrium buffer at room temperature. The terminal deoxynucleotidyl transferase (TdT) enzyme was then dropped onto the slides and was reacted in a wet chamber for $1 \mathrm{~h}$. After washing three times with PBS, the slides were treated with a permeabilization solution $(0.1 \%$ Triton $X-100,0.1 \%$ sodium citrate) at $4^{\circ} \mathrm{C}$ for $2 \mathrm{~min}$ and then washed. A TUNEL reaction solution (TdT, fluorescein-dUTP mixed solution) was used to label the chamber where the humidity was maintained at $37^{\circ} \mathrm{C}$ for $60 \mathrm{~min}$, and then the slides were washed and observed with a fluorescence microscope.

\section{Statistical analyses}

Statistical comparisons between the treated and untreated groups were performed using a two-tailed Student t-test for paired samples. The results are expressed as mean \pm standard deviation from at least three independent experiments, and ${ }^{*} p<0.05,{ }^{* *} p<0.01$, and ${ }^{* * *} p$ $<0.001$ were considered statistically significant.

\section{RESULTS}

\section{Visual and instrumental comparison of CET and RET types}

After the application of CRMRF, visual evaluation was conducted by an expert as a safety evaluation to check for damage to or changes on the outside of the skin. For each condition, no significant difference was visually observed from the normal skin surface, and no external change was observed even on day 1 , the day after the test. Changes in the skin, eyes, mucous membrane, breathing, acclimation, autonomic and central nervous systems, behavioral patterns, gait, and posture were observed. No problems occurred throughout the study. Both CET and RET were found to be safe, as they did not cause significant heat damage or side effects on the skin under each condition.

For skin care improvement using a high-frequency stimulator, the temperature of the skin surface should be determined. Therefore, in this study, the skin surface temperature was measured using a thermal camera, in accordance with the CRMRF treatment condition. The maximum temperature was identified as $40.5^{\circ} \mathrm{C}$, and was confirmed to be safe, without any macroscopic problems, even with heat sensation on the skin immediately after use (Fig. 2). Furthermore, the safety of all three probes used in the study device was confirmed.

\section{In vivo and ex vivo temperature measurement}

The heat energy generated in the RF region was transferred from the surface of the skin to the subcutaneous fat at a selective temperature of $42-47^{\circ} \mathrm{C}$. To experimentally confirm that thermolysis was progressing well, the temperatures of the skin surface and the fat layer were measured. Measurement of the pigs' skin surface temperatures using a ThermoCamera revealed that the skin surface temperature was $42-47^{\circ} \mathrm{C}$ when the test conditions for each group were applied. Under all conditions, the tests were conducted without thermal burns on the skin surface.

In addition, ex vivo measurement of the temperature change of the full skin layer cross-section using a ThermoCamera device revealed that after the application of CRMRF using the CET probe, the temperature changed only on the skin surface, preventing deeper heat transfer. On the other hand, the temperature change after application using the RET probe gradually moved from the skin surface to the fat layer and the heat was effectively absorbed by the tissue.

As a result, both the CET and RET methods used in the experiment in this study were confirmed to have generated a temperature that would not cause thermal damage on the skin surface.

\section{Evaluation of lipid profiles and hematological parameters}

Serum was separated from the blood collected at 0, 2, 4, $8,12,24$, and $72 \mathrm{~h}$ for each condition after the application of CRMRF and the levels of lipids (free fatty acids (FFA), TG, T-CHO, HDL-CHO, LDL-CHO, and phospholipids) were determined (Fig. 3).

No renal or thymus dysfunction, liver dysfunction, or lipid level abnormalities were found, and changes in the values remained within a safe range. At 24 and $72 \mathrm{~h}$, it was confirmed that most of the parameters measured had returned to their pretreatment values. Considering that the cholesterol levels for patients in the RET group were higher than for those in the CET group, RET was considered more appropriate for treatments aimed at fat reduction. Overall, the values were confirmed to be within the normal range, suggesting that the application of CRMRF (CET and RET) is viable and safe for subcutaneous fat decomposition.

\section{Ultrasound imaging and Histological change measurement for efficacy evaluation}

Ultrasound imaging was performed at baseline (0), immediately after the treatment, and on days 1, 7, 15, 30, 60, 
A

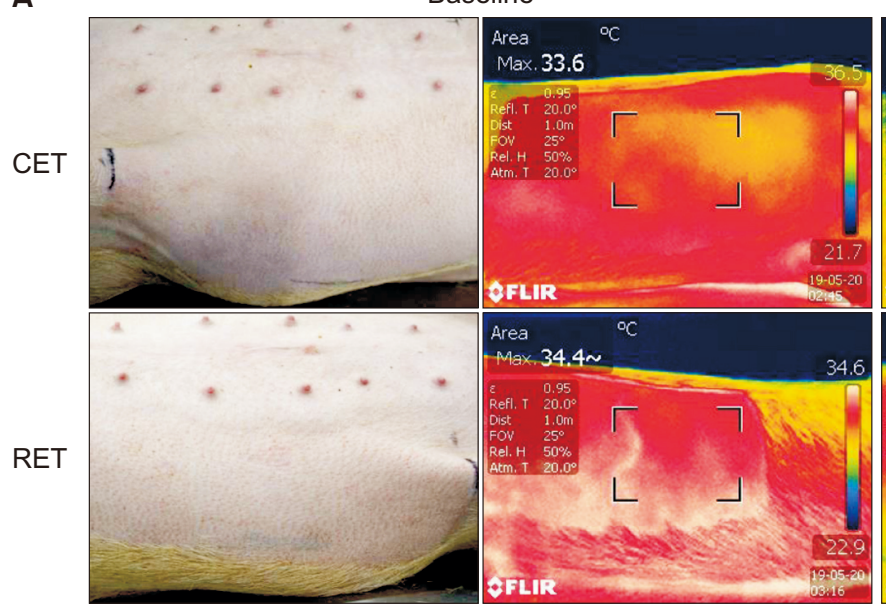

B

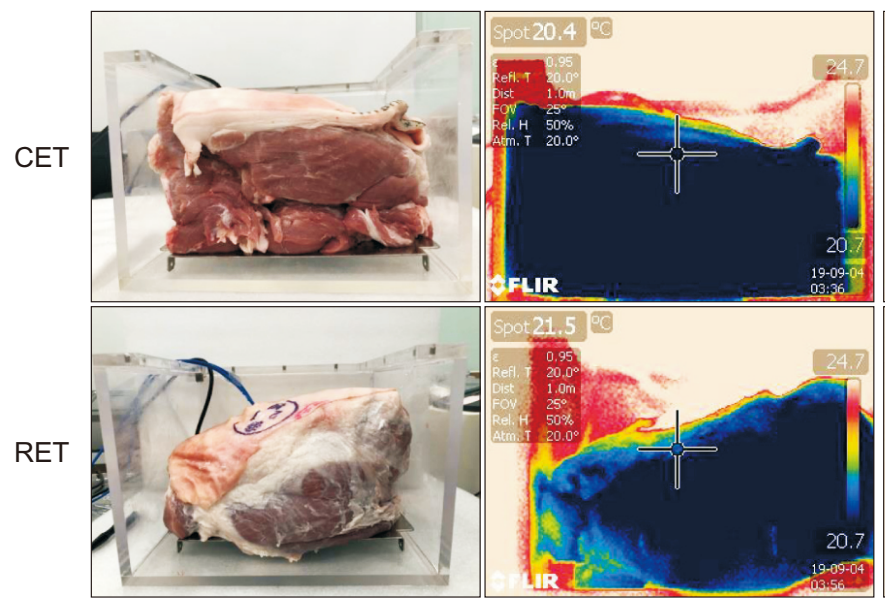

$5 \min$

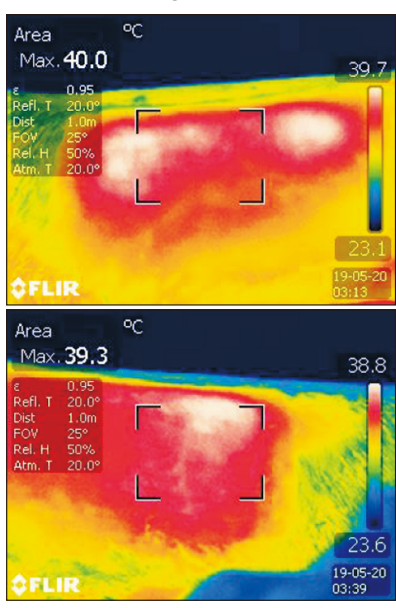

$5 \min$

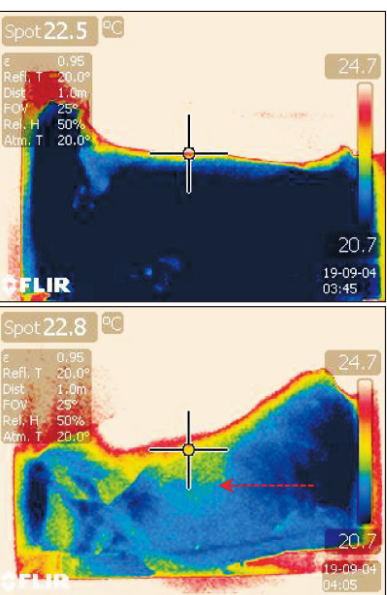

$10 \mathrm{~min}$

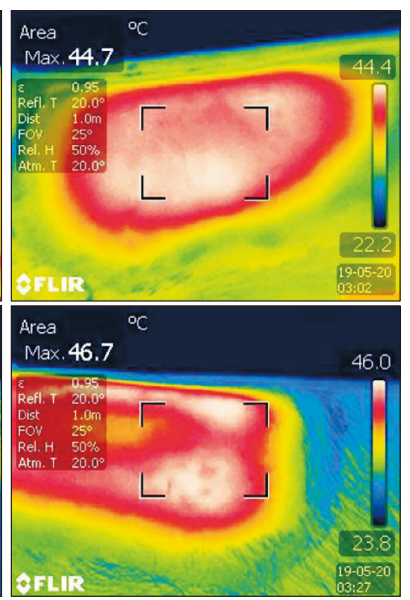

10 min

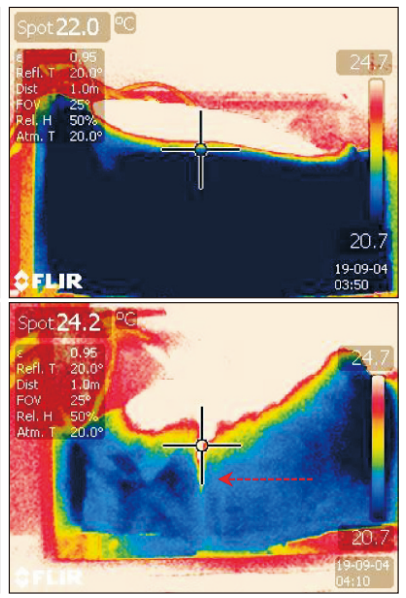

Fig. 2. Thermal profile changes in porcine skin temperature with CET and RET types of CRMRF. (A) In vivo skin surface temperature changes during the application of CRMRF (CET and RET applicator) and after the removal of the device immediately after the application. The energy of CRMRF transmits heat only to the skin surface under the applicator, and it was confirmed that around $42-47^{\circ} \mathrm{C}$ heat is generated, a safe temperature that does not cause skin damage. (B) For each energy generation during the application of the CET and RET types of CRMRF, heat transfer to the tissue was evaluated after generating heat on the surface of the ex vivo pig skin tissue layer.
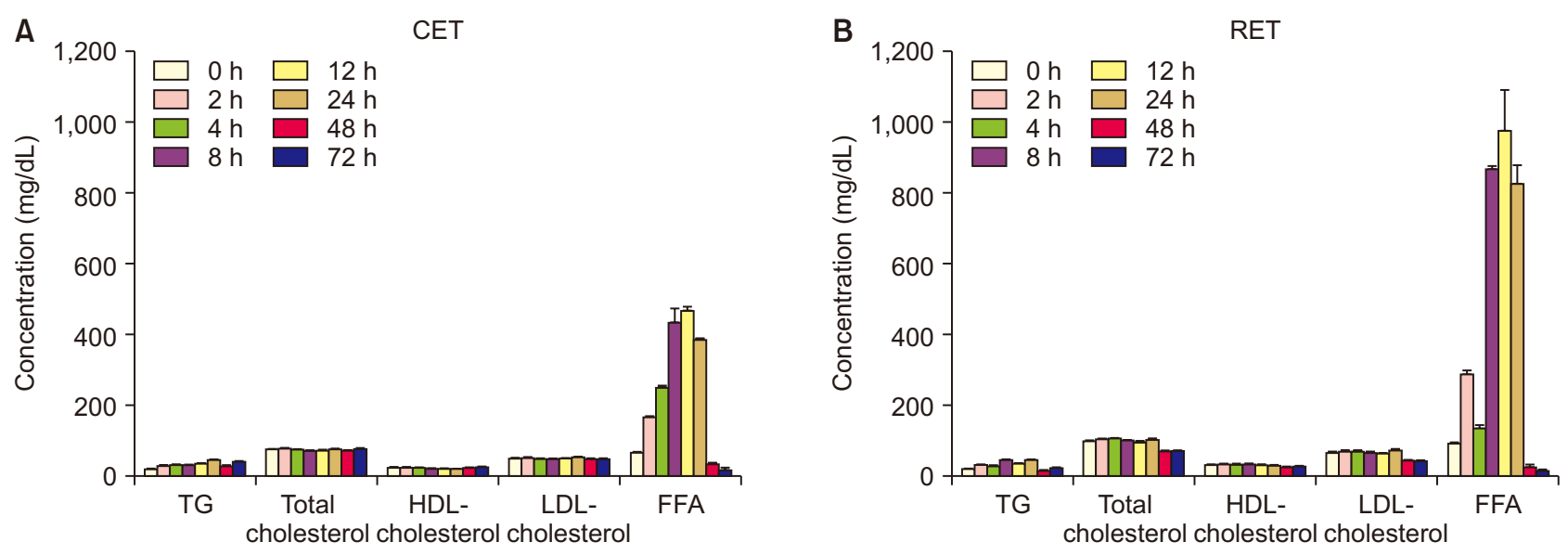

Fig. 3. Posttreatment changes in serum lipid levels. The blood was analyzed by separating the serum from the blood collected at $0,2,4,8,12,24$, and $72 \mathrm{~h}$ after applying (A) CET and (B) RET types of CRMRF. Levels of serum lipids, including total cholesterol, LDL, HDL, TG, and FFA, were examined. 
and 90 to confirm the changes in the subcutaneous fat layer after the application of CRMRF. The energy delivered to the entire abdomen was delivered to the subcutaneous fat layer, and only the adipocytes in the area were reduced (Fig. 4). In particular, immediately after the treatment, thickness and shape changes of the subcutaneous fat layer (hyperechoic pattern change) appeared. Especially, the results of the RET revealed that they are more effective in reducing the deep fat layer than the CET.

After application of CET and RET to the treatment site, a skin histopathologic examination was performed us- ing 8-mm punch biopsy. The collected skin tissues were soaked in a 10\% formalin fixative, and paraffin blocks were created for $\mathrm{H} \& \mathrm{E}$ and Oil red $\mathrm{O}$ staining to determine whether a sufficient amount of the heat generated from the RF probe was delivered to the fat layer to reduce the subcutaneous fat (Fig. 5).

H\&E staining showed sporadic coagulo-necrotic lesions in the adipose tissue immediately after the treatment, and the cell membranes of the adipocytes were unstable or destroyed. As time passed after the treatment, the fibrotic septa overlapped in the adipose tissue. After 30 days, the
A

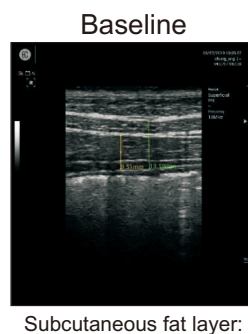

$13.19 \mathrm{~mm}$

Deep fat layer: $9.51 \mathrm{~mm}$

Day 15

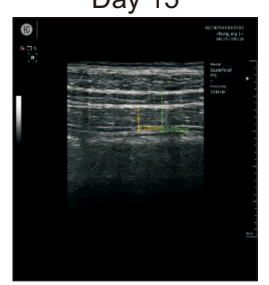

Subcutaneous fat layer: $10.69 \mathrm{~mm}$ Deep fat layer: $6.28 \mathrm{~mm}$

B

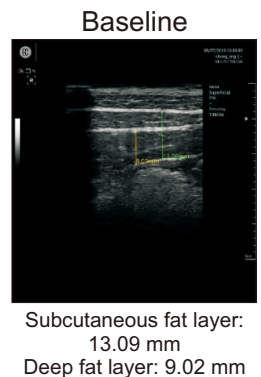

Day 15

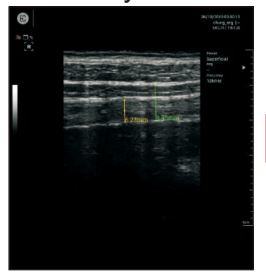

Subcutaneous fat layer: $9.95 \mathrm{~mm}$

Deep fat layer: $6.32 \mathrm{~mm}$

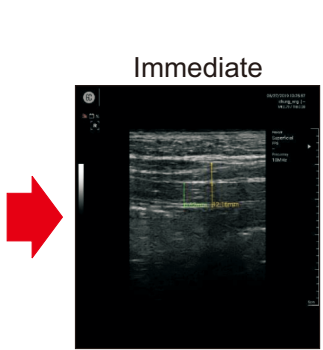

Subcutaneous fat layer: $12.16 \mathrm{~mm}$ Deep fat layer: $6.62 \mathrm{~mm}$

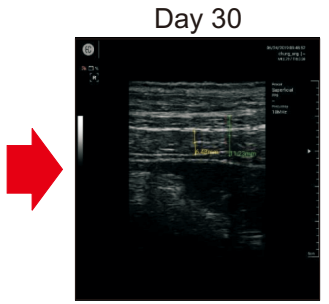

Subcutaneous fat layer: $11.23 \mathrm{~mm}$ Deep fat layer: $6.48 \mathrm{~mm}$
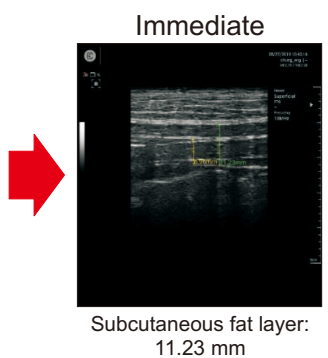
Deep fat layer: $6.96 \mathrm{~mm}$

Day 30

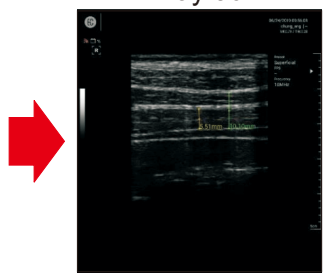

Subcutaneous fat layer: $10.1 \mathrm{~mm}$ Deep fat layer: $5.51 \mathrm{~mm}$
$\underline{\mathrm{CET}}$

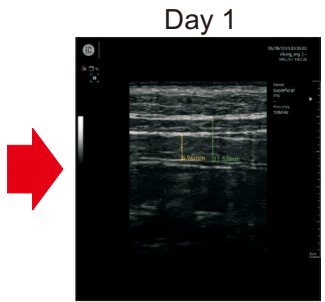

Subcutaneous fat layer: $11.52 \mathrm{~mm}$ Deep fat layer: $6.96 \mathrm{~mm}$

Day 60

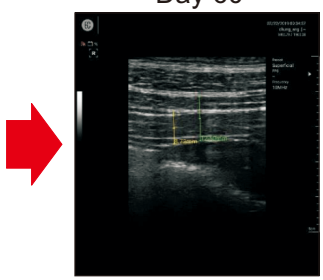

Subcutaneous fat layer: $12.5 \mathrm{~mm}$ Deep fat layer: $8.72 \mathrm{~mm}$

RET

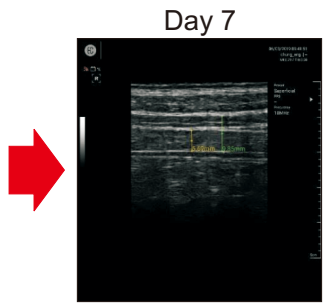

Subcutaneous fat layer: $9.85 \mathrm{~mm}$ Deep fat layer: $5.69 \mathrm{~mm}$

Day 90

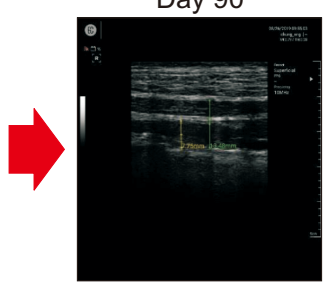

Subcutaneous fat layer: $13.48 \mathrm{~mm}$ Deep fat layer: $7.75 \mathrm{~mm}$
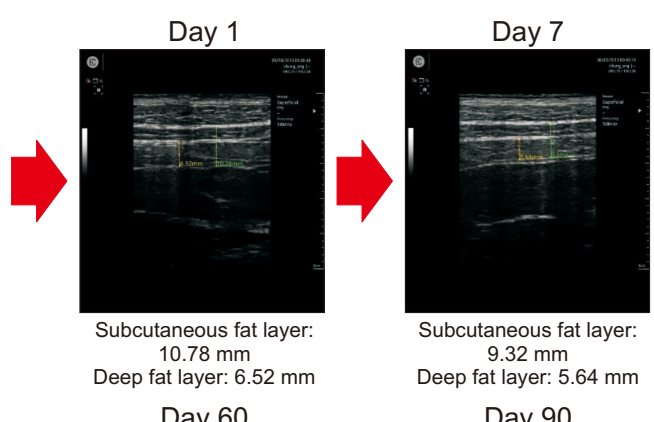

Day 60

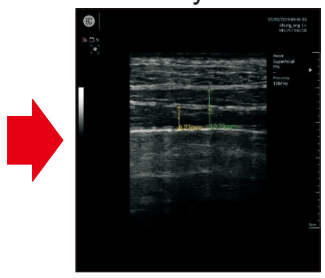

Subcutaneous fat layer: $10.79 \mathrm{~mm}$ Deep fat layer: $6.27 \mathrm{~mm}$

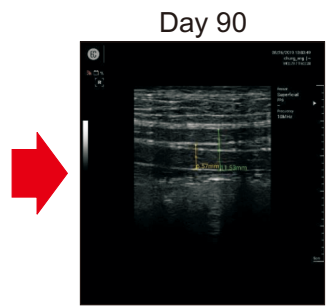

Subcutaneous fat layer: $11.53 \mathrm{~mm}$ Deep fat layer: $6.57 \mathrm{~mm}$
Fig. 4. Changes in fat layer thickness, determined with ultrasound measurement. Ultrasound images at baseline, immediately after treatment with CRMRF, and on days 1, 7, 15, 30, 60, and 90 after treatment using (A) CET and (B) RET. 

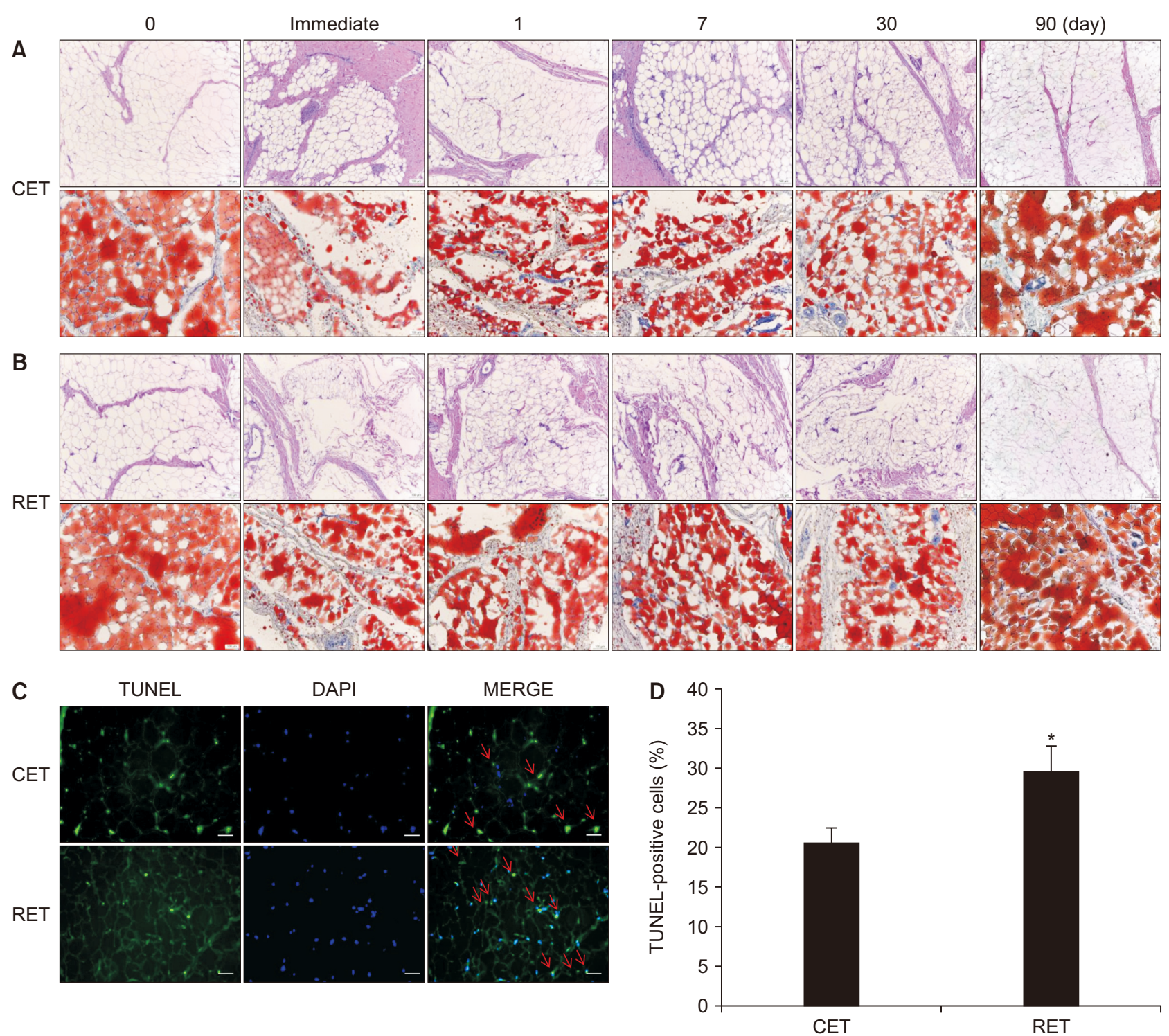

Fig. 5. Effects of CRMRF application on inflammatory responses and lipid reduction in porcine skin. Pictures taken after tissue staining (A) H\&E, (B) Oil red O, (C) TUNEL assay, (D) Quantitative TUNEL assay for the abdominal skin biopsied tissues at baseline, immediately after the treatment with CRMRF, and on days 30 after the treatment. Green; apoptotic cells, blue; cell nuclei. Original magnification: $\times 100$.

diaphragm gradually disappeared and was normalized over the course of recovery. Cell change appeared rapidly following treatment with RET and adipocyte destruction was found in all four conditions. There was a significant difference, however, in the change rate of the adipocytes close to the dermis and in that of the fat layer below.

Oil red 0 staining revealed a pattern of damage in the adipocytes due to heat and recovery was confirmed. In particular, with RET, the pattern by which all the adipocytes underwent macrophage infiltration until day 15 and entered into the process of programmed cell death showed a more effective outcome than that of CET.

\section{CRMRF induced increased apoptosis in vivo}

After the application of CRMRF at the treatment site, TUNEL staining was performed with the tissue section peeled from the same paraffin block as above to determine if selective thermolysis was adequately transmitted to the adipocytes and if the apoptosis process was completed in the adipocytes. The most prominent aspect of the cellular morphological changes early in apoptosis was the DNA cleavage of the cell nucleus. Through the TUNEL method, the end of the 3'-hydroxy group for one of the DNA segments in the apoptotic cells was observed. These findings suggested that in all the tissues to which 
each condition was applied, the cell walls of the adipocytes were recovered and the thermal energy was better delivered with RET than with CET at 30 days.

\section{DISCUSSION}

Subcutaneous fat reduction using $448-\mathrm{kHz}$ CRMRF in a porcine model was evaluated in this study and the findings confirmed the significant induction of apoptosis signaling. The results obtained from this preclinical study are novel, as to date, similar studies have not been conducted.

Tissue behaves as a semiconductor, providing resistance to the passage of electric energy, and this is translated to an increase in temperature. The therapeutic action of CRMRF depends on this increase in temperature but also on the increase of the potential energy of the cellular membranes. Recently, a system for capacitive and resistive energy transfer was developed to function in the RF range of $0.4-0.8 \mathrm{MHz}$ with the capability of reaching deep tissues without damaging superficial tissues.

Since the data in the literature are encouraging, we wanted to evaluate the efficacy of hyperthermia in osteoarthritis following the clinical response using objective and subjective data. ${ }^{22}$ Notwithstanding the abundance of equipment currently used in physiotherapy, there are various pathological, tendinous, and muscular-enthesic processes that are particularly resistant to treatment and limit the possibility of engaging in competitive and noncompetitive sports.

The efficacy of subcutaneous fat reduction using hyperthermic methods is based on the intensification of particular phases of the inflammatory process that accompanies increases in temperature. The vasodilatation obtained with heat induces an increase in the exchange of substances expressed with an increase in drainage at the inflamed site, removal of waste, and improvement of tissue perfusion with an increase in the local influx of cells involved in reparative processes. Our findings suggested that subcutaneous fat reduction with CRMRF using CET and RET is safe and effective. This system, based on the $448-\mathrm{kHz}$ frequency, which is the key to active cell therapy, facilitates biostimulation, stimulates microcirculation, and promotes metabolic hyperactivation, while respecting cell physiology. ${ }^{4,19,20}$

This study evaluated the safety and efficacy of CRMRF for subcutaneous fat reduction by applying CET and RET to a porcine model. Neither the CET nor RET method caused skin surface damage, dermal inflammatory or thermal reactions, or tissue necrosis on the skin surface.
In addition, hematological analysis for the application of CRMRF showed that the liver function and lipid levels were changed within the normal range, and this did not have a toxic effect on the kidneys or thymus. Moreover, after treatment with CRMRF using CET and RET, changes in the subcutaneous fat layer in the abdomen were measured via ultrasound at different times for comparison purposes.

The thermolysis action of CRMRF was adequately transmitted to the target fat layer, leading to a temperature change to over $40^{\circ} \mathrm{C}$ in the fat layer. On the skin surface, an approximately $42^{\circ} \mathrm{C}$ temperature was confirmed. In addition, it was confirmed that the subcutaneous fat layer thickness was reduced by using the ultrasonic device for each application period 10 , immediately after the treatment, and on days 1, 7, 15,30,60, and 90 after the treatment).

After treatment with CRMRF using CET and RET, the change in the thickness of the subcutaneous fat layer in the abdomen was measured via ultrasound at different times for comparison purposes.

To verify the treatment effect of the two conditions, skin pathological biopsy was used to confirm the inflammatory response, and $\mathrm{H} \& \mathrm{E}$ and Oil red $\mathrm{O}$ staining were used to confirm the effect of fat reduction. In the search for potential alternative candidate modalities for lipolysis, the RF device for effective reduction of the subcutaneous fat layer was investigated as a promising candidate using in vivo studies.

In our study, after treatment with CRMRF using CET and RET, histological changes in the subcutaneous fat layer in the abdomen were observed and compared through H\&E (inflammatory cell identification), Oil red 0 (adipocyte identification), and TUNEL staining lapoptosis confirmation). In particular, the TUNEL staining studies showed that the CET- and RET-treated groups had markedly increased apoptotic cells compared to the control group. Apoptosis is characterized by strong DNA compaction and a high degree of DNA fragmentation. Tissue fixation, i.e., protein and DNA crosslinking and denaturation, hinders TUNEL reagent access to the $3^{\prime} \mathrm{OH}$ recessed ends. ${ }^{23}$ These results suggest that CRMRF alters certain functions that stimulate RF energy-induced apoptotic cell death. Additionally, the results presented in this study may help elucidate the processes at both the membrane and DNA levels involved in adipocyte damage following their exposure to thermal energy by the CET and RET types of CRMRF.

Tissue staining confirmed that fibrotic septa occurred in the adipose tissues at the treatment sites for all groups, 
which indirectly confirmed that the mast cells became active, thereby inducing adipocyte death. Moreover, the Oil red 0 staining showed a pattern of damage by heat and recovery of the adipocytes. Additionally, TUNEL staining was performed to check the apoptosis process in the adipocytes, and the results indicated that RET was more effective than CET in this regard.

\section{CONCLUSION}

In conclusion, this study was conducted to obtain data on the safety and performance of CRMRF for subcutaneous fat reduction, and a comparative test was conducted on the RET and CET types of RF stimulators. Further welldesigned trials to confirm the results of the present case study are needed.

\section{ACKNOWLEDGEMENT}

This research was supported by the Chung-Ang University Research Scholarship Grants in 2019.

\section{CONFLICT OF INTEREST}

The authors declare that they have no conflicts of interest to disclose.

\section{REFERENCES}

1. Seidell JC, Deurenberg P, Hautvast JG. Obesity and fat distribution in relation to health--current insights and recommendations. World Rev Nutr Diet 1987;50:57-91.

2. Pasquali R, Casimirri F, Cantobelli S, Melchionda N, Morselli Labate AM, Fabbri R, et al. Effect of obesity and body fat distribution on sex hormones and insulin in men. Metabolism 1991;40:101-4.

3. Caterson ID, Gill TP. Obesity: epidemiology and possible prevention. Best Pract Res Clin Endocrinol Metab 2002;16:595610.

4. Kennedy J, Verne S, Griffith R, Falto-Aizpurua L, Nouri K. Noninvasive subcutaneous fat reduction: a review. J Eur Acad Dermatol Venereol 2015;29:1679-88.

5. Sasaki GH, Abelev N, Tevez-Ortiz A. Noninvasive selective cryolipolysis and reperfusion recovery for localized natural fat reduction and contouring. Aesthet Surg J 2014;34:420-31.

6. Guth F, Bitencourt S, Bedinot C, Sinigaglia G, Tassinary JAF. Immediate effect and safety of HIFU single treatment for male subcutaneous fat reduction. J Cosmet Dermatol 2018;17:3859.

7. Downie J, Kaspar M. Contactless abdominal fat reduction with selective RFTM evaluated by Magnetic Resonance Imaging (MRI): case study. J Drugs Dermatol 2016;15:491-5.

8. Caruso-Davis MK, Guillot TS, Podichetty VK, Mashtalir N, Dhurandhar NV, Dubuisson O, et al. Efficacy of low-level laser therapy for body contouring and spot fat reduction. Obes Surg 2011;21:722-9.

9. Gorgu M, Gökkaya A, Karabekmez FE, Aytar O, Kızılkan J, Karanfil E, et al. Effects of device variables to radiofrequency (RF) applications. J Cosmet Laser Ther 2019;21:364-71.

10. Black DR, Heynick LN. Radiofrequency (RF) effects on blood cells, cardiac, endocrine, and immunological functions. Bioelectromagnetics 2003;Suppl 6:S187-95.

11. Hong K, Georgiades C. Radiofrequency ablation: mechanism of action and devices. J Vasc Interv Radiol 2010;2118 Suppl):S179-86.

12. Helfrich YR, Sachs DL, Voorhees JJ. Overview of skin aging and photoaging. Dermatol Nurs 2008;20:177-83; quiz 84.

13. Boisnic S, Divaris M, Nelson AA, Gharavi NM, Lask GP. A clinical and biological evaluation of a novel, noninvasive radiofrequency device for the long-term reduction of adipose tissue. Lasers Surg Med 2014;46:94-103.

14. Dunbar SW, Goldberg DJ. Radiofrequency in cosmetic dermatology: an update. J Drugs Dermatol 2015;14:1229-38.

15. Park YJ, Jo YW, Bang SI, Kim HJ, Lim SY, Mun GH, et al. Radiofrequency volume reduction of gastrocnemius muscle hypertrophy for cosmetic purposes. Aesthetic Plast Surg 2007;31:5361.

16. Lolis MS, Goldberg DJ. Radiofrequency in cosmetic dermatology: a review. Dermatol Surg 2012;38:1765-76.

17. Sadick NS, Mulholland RS. A prospective clinical study to evaluate the efficacy and safety of cellulite treatment using the combination of optical and RF energies for subcutaneous tissue heating. J Cosmet Laser Ther 2004;6:187-90.

18. Spottorno J, Gonzalez de Vega C, Buenaventura M, Hernando A. Influence of electrodes on the $448 \mathrm{kHz}$ electric currents created by radiofrequency: a finite element study. Electromagn Biol Med 2017;36:306-14.

19. Hernández-Bule ML, Martínez-Botas J, Trillo MÁ, Paíno CL, Úbeda A. Antiadipogenic effects of subthermal electric stimulation at $448 \mathrm{kHz}$ on differentiating human mesenchymal stem cells. Mol Med Rep 2016;13:3895-903.

20. Hernández-Bule ML, Paíno CL, Trillo MÁ, Úbeda A. Electric stimulation at $448 \mathrm{kHz}$ promotes proliferation of human mesenchymal stem cells. Cell Physiol Biochem 2014;34:1741-55.

21. Kwon TR, Im S, Jang YJ, Oh CT, Choi EJ, Jung SJ, et al. Improved methods for evaluating pre-clinical and histological effects of subcutaneous fat reduction using high-intensity focused ultrasound in a porcine model. Skin Res Technol 2017;23:194-201.

22. Kumaran B, Watson T. Treatment using $448 \mathrm{kHz}$ capacitive 
resistive monopolar radiofrequency improves pain and function in patients with osteoarthritis of the knee joint: a randomised controlled trial. Physiotherapy 2019;105:98-107.
23. Portt L, Norman G, Clapp C, Greenwood M, Greenwood MT. Anti-apoptosis and cell survival: a review. Biochim Biophys Acta 2011;1813:238-59. 